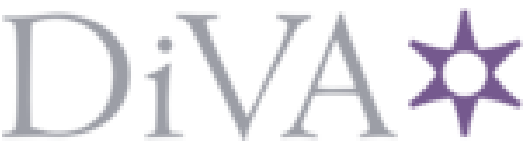

http://www.diva-portal.org

Postprint

This is the accepted version of a paper presented at IEEE Sensors 2017, 29 Oct - O1 Nov 2017, Glasgow, United Kingdom.

Citation for the original published paper:

Halvorsen, K., Olsson, F. (2017)

Robust tracking of periodic motion in the plane using inertial sensor data.

In: Proceedings of IEEE Sensors 2017 Glasgow, Scotland

N.B. When citing this work, cite the original published paper.

Permanent link to this version:

http://urn.kb.se/resolve?urn=urn:nbn:se:uu:diva-334069 


\section{Robust Tracking of Periodic Motion in the Plane using Inertial Sensor Data}

\author{
Kjartan Halvorsen \\ Department of Information Technology \\ Uppsala University, Sweden \\ and ITESM, Campus Estado de Mexico \\ Email: kjartan.halvorsen@it.uu.se
}

\author{
Fredrik Olsson \\ Department of Information Technology \\ Uppsala University, Sweden \\ Email: fredrik.olsson@it.uu.se
}

\begin{abstract}
We propose a robust method for estimating the orientation and displacement of an inertial measurement unit undergoing planar periodic motion. Such movements is a common approximation to human gait and running. We formulate the problem introducing a sparse vector of outlier errors and 11regularization. The problem thus becomes robust to outliers in the data. The problem can be rewritten as a quadratic programming problem which can be solved efficiently using existing software for convex optimization.
\end{abstract}

\section{INTRODUCTION}

MEMS inertial sensors nodes containing a 3D accelerometer, gyro and magnetometer (IMUs) are increasingly being used for quantitative study of human motion, but integrating the sensor data to obtain orientation and displacement causes drift. The drift in orientation can be reduced by available absolute measurements of the directions of the magnetic field and the gravitational acceleration. However, absolute measurements of position are typically not available, except in special cases [1].

Many human movements are periodic in nature, in particular locomotion. An IMU attached to a body segment will then return to the same orientation at the end of a period. We have previously developed a method which makes use of this assumption of periodicity in order to eliminate the drift [2]. In this paper, we modify this method for the particular case of planar motion. Pre-processing the sensor data allows us to formulate the problem as a robust optimization problem, which is less sensitive to outliers. The method is evaluated on data from a treadmill-walking experiment.

\section{MODEL}

\section{A. Kinematics}

We consider the movement of a single rigid body segment in a vertical plane, typically the sagittal plane (symmetry plane) of a walking or running human. The orientation is described by the angle $\phi(t)$ and the position in a global coordinate system by $p(t)=\left[\begin{array}{ll}p_{x}(t) & p_{z}(t)\end{array}\right]$. We define three coordinate systems, one whose axes align with the accelerometer triad of the IMU denoted by subscript $S$, one whose axes are fixed in the sensorattached body segment denoted by subscript $B$ and a global earth-fixed system denoted by subscript $G$. The y-axes of the $B$ and $G$ frames are normal to the plane of motion. When the body segment is in a reference pose, then $\phi=0$ and both the $z_{B}$ and $z_{G}$ vectors coincide with the vertical direction. The direction normal to the plane of motion is assumed to be known in the $S$-frame, either by attaching the IMU carefully in a specific orientation with respect to the anatomy, or by estimating the direction from sensor measurements [3]. Thus the transformation between the $S$ - and $B$-frames is known, and the transformation between the $B$-frame and $G$-frame is described by the angle $\phi(t)$.

\section{B. Parameterizing the periodic motion}

Periodic motion implies

$$
\phi(t+T)=\phi(t), \quad \text { and } \quad p(t+T)=p(t)
$$

for some value of $T$ called the period of the movement. Let $\Omega=\frac{1}{T}$ be the base frequency of the movement. The periodic movement is modeled as the truncated Fourier series with $M$ periodic components

$$
\begin{aligned}
\phi(t) & =\alpha_{\phi 0}+\sum_{n=1}^{M} \alpha_{\phi n} \cos (n \Omega t)+\sum_{n=1}^{M} \beta_{\phi n} \sin (n \Omega t) \\
p_{x}(t) & =\sum_{n=1}^{M} \alpha_{x n} \cos (n \Omega t)+\sum_{n=1}^{M} \beta_{x n} \sin (n \Omega t)-\sum_{n=1}^{M} \alpha_{x n},
\end{aligned}
$$

and similar for $p_{z}(t)$. The sum of the coefficients for the cosines is subtracted from the model of the displacement in order to obtain a trajectory that starts at the origin.

We define the vector of unknown coefficients

$$
\begin{aligned}
\theta_{\phi} & =\left[\begin{array}{llllll}
\alpha_{\phi 0} & \cdots & \alpha_{\phi M} & \beta_{\phi 1} & \cdots & \beta_{\phi M}
\end{array}\right]^{\top} \\
\theta_{x} & =\left[\begin{array}{llllll}
\alpha_{x 1} & \cdots & \alpha_{x M} & \beta_{x 1} & \cdots & \beta_{x M}
\end{array}\right]^{\top} \\
\theta_{z} & =\left[\begin{array}{llllll}
\alpha_{z 1} & \cdots & \alpha_{z M} & \beta_{z 1} & \cdots & \beta_{z M}
\end{array}\right]^{\top}
\end{aligned}
$$

with which the orientation and displacement can be written

$$
\begin{gathered}
\phi(t)=\left[\begin{array}{ll}
1 & A(t)
\end{array}\right] \theta_{\phi}, \\
p_{x}(t)=(A(t)-C) \theta_{x}, \quad p_{z}(t)=(A(t)-C) \theta_{z},
\end{gathered}
$$

where

$A(t)=\left[\begin{array}{llllll}\cos (\Omega t) & \cdots & \cos (M \Omega t) & \sin (\Omega t) & \cdots & \sin (M \Omega t)\end{array}\right]$

and $C=\left[\begin{array}{ll}\mathbf{1}_{M} & 0\end{array}\right]$. The time derivatives become

$$
\begin{array}{r}
\dot{\phi}(t)=\left[\begin{array}{ll}
0 & \dot{A}(t)
\end{array}\right] \theta_{\phi}, \\
\ddot{p}_{x}(t)=\ddot{A}(t) \theta_{x}, \quad \ddot{p}_{z}(t)=\ddot{A}(t) \theta_{z} .
\end{array}
$$




\section{Pre-processing of the data}

We consider a sequence of $N$ data samples of gyro data, $w_{S}(t)$, and acceleration data, $a_{S}(t)$. The data contain several periods of the movement, and the start of each period is detected from the data based on particular data patterns. In gait and running, for instance, the acceleration will show a large peak when the foot make contact with the ground. The following processing of the data is motivated by the goal to obtain linear relationships between the data and the parameters of orientation- and displacement models (5) - (6).

Some of the $N$ acceleration samples have magnitude sufficiently close to the gravitational acceleration $g$ to be used as inclinometer measurements

$$
\left\{\tau_{1}, \tau_{2}, \ldots, \tau_{k}\right\}=\left\{t \mid \operatorname{abs}\left(\left\|a_{S}(t)\right\|_{2}-g\right)<\epsilon\right\} .
$$

In our experiments we found $\epsilon=0.05 g$ to work well. The inclination measurements are stacked to form

$$
y_{i n c}^{\top}=\left[\begin{array}{llll}
y_{i n c}\left(\tau_{1}\right) & y_{i n c}\left(\tau_{2}\right) & \cdots & y_{i n c}\left(\tau_{k}\right)
\end{array}\right],
$$

where $y_{\text {inc }}\left(\tau_{i}\right):=\operatorname{atan} 2\left(-a_{B, x}\left(\tau_{i}\right), a_{B, z}\left(\tau_{i}\right)\right)$ and using the known transformation between the $S$ and $B$ frames.

For the gyro data we make use of the assumption that since the segment returns to the same orientation after a period, the angular velocity must be zero-mean over each period. The gyro-data are thus projected onto the axis of rotation $y_{B}$ and then detrended

$$
y_{\text {gyr }}(t):=\operatorname{detrend}_{\text {const }}\left(w_{S}(t) \cdot y_{B}\right),
$$

where the function detrend $\mathrm{d}_{\text {const }}$ removes a constant trend over each period.

The pre-processing of the accelerometer data depends on an available estimate of the angle $\phi(t)$, so that the acceleration measurements $a_{S}(t)$ can be rotated into the global coordinate system. Since the displacement is assumed to be periodic and hence zero at the beginning and end of each period, the acceleration expressed in the global coordinate system must have zero linear trend. The data are processed as

$$
\begin{aligned}
& y_{a x}(t):=\text { detrend }_{\operatorname{lin}}\left(a_{G}(t) \cdot x_{G}\right) \\
& y_{a z}(t):=\text { detrend }_{\operatorname{lin}}\left(a_{G}(t) \cdot z_{G}\right),
\end{aligned}
$$

where $a_{G}(t)$ is the acceleration vector in the global frame, and the function detrend $d_{\text {lin }}$ removes a linear trend over each period of data.

\section{Signal model}

The angular velocity in the plane of motion is described by the truncated Fourier series model (7) plus two noise terms

$$
\begin{aligned}
y_{g y r}(t) & =\left[\begin{array}{ll}
0 & \dot{A}(t)
\end{array}\right] \theta_{\phi}+e_{g y r}(t)+v_{g y r}(t) \\
& =A_{g y r}(t) \theta_{\phi}+e_{g y r}(t)+v_{g y r}(t),
\end{aligned}
$$

where $e_{g y r}(t)$ is an outlier term which is most often zero and $v_{g y r}(t)$ is white Gaussian noise. Similarly, the inclinometer measurements are modeled as

$$
\begin{aligned}
y_{i n c}(t) & =\left[\begin{array}{ll}
1 & A(t)
\end{array}\right] \theta_{\phi}+e_{i n c}(t)+v_{i n c}(t) \\
& =A_{i n c}(t) \theta_{\phi}+e_{i n c}(t)+v_{i n c}(t),
\end{aligned}
$$

and the acceleration in the global plane of motion as

$$
\begin{aligned}
& y_{a x}(t)=\ddot{A}(t) \theta_{x}+e_{x}(t)+v_{x}(t) \\
& y_{a z}(t)=\ddot{A}(t) \theta_{z}+e_{z}(t)+v_{z}(t) .
\end{aligned}
$$

The sparse noise terms are included to describe outliers in the data and modeling errors, and will make the estimates more robust to such errors than a model with only Gaussian noise terms. In particular, the inclination data $y_{\text {inc }}$ are prone to significant outliers whenever the acceleration of the rigid body is large and directed such that the accelerometer measurement has magnitude close to $g$.

\section{ESTIMATING THE ORIENTATION AND DISPLACEMENT}

Stacking the signal models (12) and (13) for all samples, we obtain the data model

$$
\begin{aligned}
y_{\phi} & =\left[\begin{array}{l}
A_{g y r} \\
A_{i n c}
\end{array}\right] \theta_{\phi}+\left[\begin{array}{l}
e_{g y r} \\
e_{i n c}
\end{array}\right]+\left[\begin{array}{l}
v_{g y r} \\
v_{i n c}
\end{array}\right] \\
& =A_{\phi} \theta_{\phi}+e_{\phi}+v_{\phi} .
\end{aligned}
$$

where $e_{g y r}$ and $e_{i n c}$ are sparse vectors of outliers, and $v_{g y r}$ and $v_{\text {inc }}$ are independent Gaussian noise vectors with known covariance matrices, assumed or estimated from the data for a sensor at rest. The vector of model parameters $\theta_{\phi}$ is determined as the solution to the optimization problem

$$
\begin{aligned}
\underset{\theta_{\phi}, e_{g y r}, e_{i n c}}{\operatorname{minimize}} & \left\|A_{\phi} \theta_{\phi}+e_{\phi}-y_{\phi}\right\|_{\Sigma_{\phi}^{-1}} \\
& +\lambda_{\text {gyr }}\left\|e_{g y r}\right\|_{1}+\lambda_{\text {inc }}\left\|e_{i n c}\right\|_{1},
\end{aligned}
$$

where $\Sigma_{\phi}$ is the covariance matrix of $v_{\phi}$, and the parameters $\lambda_{g y r}$ and $\lambda_{i n c}$ are chosen so that the resulting sparse outlier vectors $e_{g y r}$ and $e_{i n c}$ have the expected number of nonzero elements. The optimization problem can be rewritten as a quadratic programming (QP) problem [4]. and solved efficiently using software for convex optimization such as cvxopt [5]

The estimate of the displacement is obtained similarly by forming the data model

$$
\begin{aligned}
& y_{a x}=A_{p} \theta_{x}+e_{x}+v_{x}, \\
& y_{a z}=A_{p} \theta_{z}+e_{z}+v_{z},
\end{aligned}
$$

where

$$
A_{p}=\left[\begin{array}{llll}
\ddot{A}^{\top}(1) & \ddot{A}^{\top}(2) & \cdots & \ddot{A}^{\top}(N)
\end{array}\right]^{\top},
$$

the sparse vectors $e_{x}$ and $e_{z}$ contain outliers, and $v_{x}$ and $v_{z}$ are Gaussian measurement noise.

\section{EXPERIMENTAL EVALUATION}

Data of human gait was acquired using both camera-based motion capture (ProReflex, Qualisys AB, Sweden) and inertial measurement units (x-IMU, $\mathrm{x}$-io Technologies, UK). The testsubject walked on a treadmill for several intervals of five minutes. Four cameras tracked reflective markers placed on the sides of the left ankle (lateral malleolus) and the left knee. One IMU was strapped to the shank laterally, proximal of the left ankle. One IMU was strapped laterally on the thigh. The unit vector in the direction of the knee joint axis was used as the normal vector to the plane of motion, calculated from the IMUs on the shank and the thigh using the method 


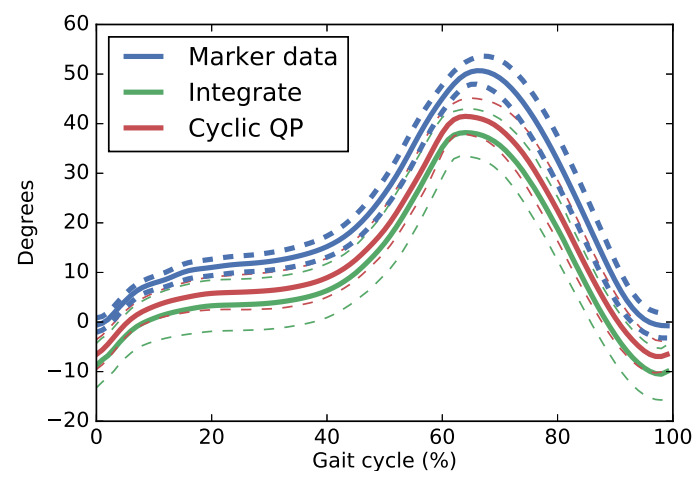

Fig. 1. Angle to the vertical for the shank segment. Solid lines are ensemble means from 41 gait cycles. Dashed lines show mean \pm 2 standard deviations. $M=16$ terms were included in the Fourier series.

proposed in [3]. The number of terms $M$ to include in the Fourier series was chosen based on the typical bandwidth of kinematics data in human gait. For the displacement model the number of terms was chosen higher, since the waveform of the vertical displacement signal for the ankle has a flat section which requires more overtones. The weighting factors $\lambda_{g y r}, \lambda_{i n c}$ were chosen such that the number of outliers in the gyro data was about $1 \%$ and the number of outliers in the inclinometer data was about $50 \%$. These values were deemed reasonable, but extensive tuning of the parameters was not performed.

As reference, the angle of the vector from the ankle- to the knee marker to the vertical direction was calculates, as well as the displacement of the ankle marker. In addition, the results of the proposed method are compared to numerical integration of the pre-processed gyro data (10) and accelerometer data (11) for each gait cycle using the trapezoidal method. A constant offset was added to the integrated gyro data to obtain a leastsquares fit to the inclinometer data (9). The estimated angle to the vertical for gait data over an interval of one minute is shown in figure 1 and the estimated displacement in the vertical and forward-backward directions are shown in figure 2.

\section{Discussion AND CONCLUSION}

Integrating IMU data to obtain orientation and displacement is known to be prone to drift, even over a short interval such as a gait cycle. However, the assumption of a periodic motion can be used to effectively eliminate the drift, as the data (properly transformed and expressed in the global coordinate system) will be zero-mean (gyro data) or zero-linear-trend (accelerometer data). The difference between the two IMUbased methods was small for this experimental evaluation, which is a little surprising, since we expected the Fourierseries to better reduce the effect of outliers in the data. In particular, the inclinometer data is prone to outliers, since the acceleration can be near $g$ in magnitude without being near vertical. In simulations (results not included) the proposed method was seen to be less sensitive to outliers than was the regular integration of sensor data. In contrast, the results from the experimental data (figures 1 and 2) show no clear difference in performance between the two methods. Probably, other sources of errors between the marker-based results and the IMU-based results are more dominating. Note also that
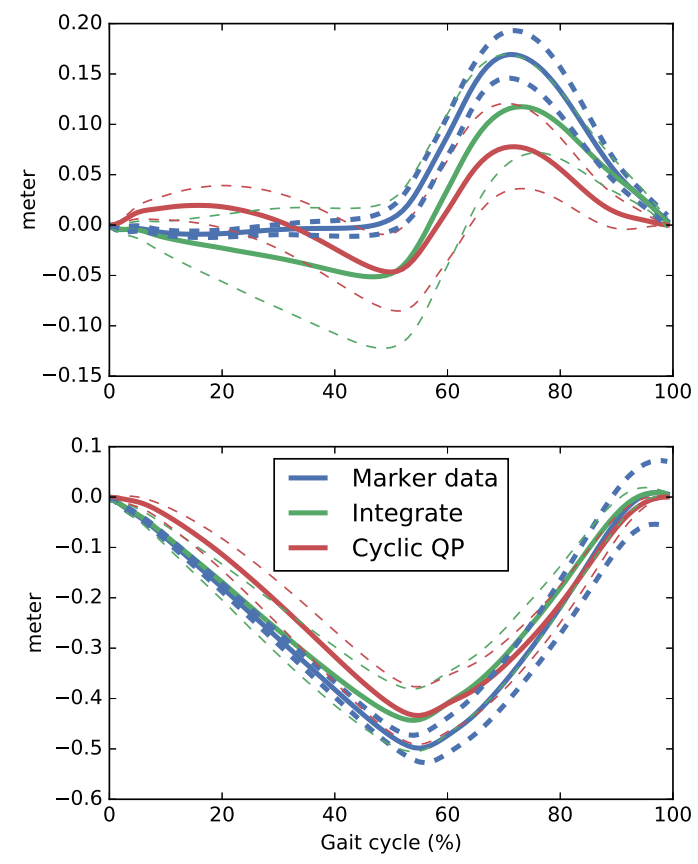

Fig. 2. Displacement of the ankle in the vertical direction (top) and forwardbackward direction (bottom). Solid lines are ensemble means from 41 gait cycles. Dashed lines show mean \pm 2 standard deviations. $M=24$ terms were included in the Fourier series.

whereas the orientation is well estimated from the IMU data, the estimate of the displacement is rather poor, particularly in the vertical direction.

This paper presents a new method for estimating planar orientation and displacement from IMUs making use of the assumption of periodic motion do eliminate drift in the estimates. Although showing promise on data with simulated outliers, the experimental evaluation on human motion does not show an improved performance over a simpler integration of pre-processed sensor data. Further tests are required in order to fully validate the method.

\section{ACKNOWLEDGMENT}

This work was supported by the Swedish Research Council under project grant 2015-05054. Thanks to Prof Ola Eiken and the Environmental Physiology group at KTH for providing validation data.

\section{REFERENCES}

[1] M. Kok, J. Hol, and T. Schön, "Indoor positioning using ultra-wideband and inertial measurements," Vehicular Technology, IEEE Transactions on, vol. PP, no. 99, pp. 1-1, 2015.

[2] K. Halvorsen and F. Olsson, "Pose estimation of cyclic movement using inertial sensor data," in Statistical Signal Processing Workshop (SSP), 2016 IEEE. IEEE, 2016, pp. 1-5.

[3] T. Seel, J. Raisch, and T. Schauer, "Imu-based joint angle measurement for gait analysis," Sensors, vol. 14, no. 4, pp. 6891-6909, 2014.

[4] S. Boyd and L. Vandenberghe, Convex optimization. Cambridge university press, 2004.

[5] M. S. Andersen, J. Dahl, and L. Vandenberghe, "Cvxopt: A python package for convex optimization, version 1.1. 6," Available at cvxopt. org, 2013. 